\title{
Survival or death: a dual role for autophagy in stress-induced pericyte loss in diabetic retinopathy
}

\author{
Dongxu Fu ${ }^{1,2} \cdot$ Jeremy Y. Yu ${ }^{1}$. Shihe Yang ${ }^{3} \cdot$ Mingyuan $\mathrm{Wu}^{3} \cdot$ Samar M. Hammad $^{4}$. \\ Anna R. Connell ${ }^{1} \cdot$ Mei Du $^{3}$ • Junping Chen ${ }^{3}$ Timothy J. Lyons ${ }^{1}$
}

Received: 31 March 2016 / Accepted: 27 June 2016 / Published online: 30 July 2016

(C) The Author(s) 2016. This article is published with open access at Springerlink.com

\begin{abstract}
Aims/hypothesis Intra-retinal extravasation and modification of LDL have been implicated in diabetic retinopathy: autophagy may mediate these effects.

Methods Immunohistochemistry was used to detect autophagy marker LC3B in human and murine diabetic and non-diabetic retinas. Cultured human retinal capillary pericytes (HRCPs) were treated with in vitro-modified heavily-oxidised glycated LDL (HOG-LDL) vs native LDL (N-LDL) with or without autophagy modulators: green fluorescent protein-LC3 transfection; small interfering RNAs against Beclin-1, c-Jun NH(2)-terminal kinase (JNK) and C/EBP-homologous protein (CHOP); autophagy inhibitor 3-MA ( $5 \mathrm{mmol} / \mathrm{l})$ and/or caspase inhibitor Z-VAD-fmk $(100 \mu \mathrm{mol} / \mathrm{l})$. Autophagy, cell viability, oxidative stress, endoplasmic reticulum stress, JNK activation, apoptosis and CHOP expression were assessed by western blots, CCK-8 assay and TUNEL assay. Finally, HOG-LDL vs N-LDL were injected intravitreally to STZ-induced diabetic vs control rats
\end{abstract}

Electronic supplementary material The online version of this article (doi:10.1007/s00125-016-4058-5) contains peer-reviewed but unedited supplementary material, which is available to authorised users.

Timothy J. Lyons

t.lyons@qub.ac.uk

1 Centre for Experimental Medicine, School of Medicine, Dentistry and Biomedical Sciences, Queen's University Belfast, 97 Lisburn Road, Belfast BT9 7BL, UK

2 Department of Immunology, Harbin Medical University, Harbin, People's Republic of China

3 Section of Endocrinology and Diabetes, University of Oklahoma Health Sciences Center, Oklahoma City, OK, USA

4 Department of Regenerative Medicine and Cell Biology, Medical University of South Carolina, Charleston, SC, USA (yielding 50 and $200 \mathrm{mg}$ protein/l intravitreal concentration) and, after 7 days, retinas were analysed for ER stress, autophagy and apoptosis.

Results Intra-retinal autophagy (LC3B staining) was increased in diabetic vs non-diabetic humans and mice. In HRCPs, 50 mg/l HOG-LDL elicited autophagy without altering cell viability, and inhibition of autophagy decreased survival. At 100-200 mg/l, HOG-LDL caused significant cell death, and inhibition of either autophagy or apoptosis improved survival. Further, 25-200 mg/1 HOG-LDL dose-dependently induced oxidative and ER stress. JNK activation was implicated in autophagy but not in apoptosis. In diabetic rat retina, $50 \mathrm{mg} / 1$ intravitreal HOG-LDL elicited autophagy and ER stress but not apoptosis; $200 \mathrm{mg} / \mathrm{l}$ elicited greater ER stress and apoptosis.

Conclusions Autophagy has a dual role in diabetic retinopathy: under mild stress (50 mg/l HOG-LDL) it is protective; under more severe stress (200 mg/l HOG-LDL) it promotes cell death.

Keywords Apoptosis - Autophagy - Diabetic retinopathy · ER stress $\cdot$ LC3B $\cdot$ ModifiedLDL $\cdot$ Oxidative stress $\cdot$ Pericytes

$\begin{array}{ll}\text { Abbreviations } \\ \text { 3-MA } & \text { 3-Methyladenine } \\ \text { ATG-5 } & \text { Autophagy-related homologue 5 } \\ \text { BRB } & \text { Blood-retinal barrier } \\ \text { CHOP } & \text { C/EBP-homologous protein } \\ \text { CQ } & \text { Chloroquine } \\ \text { eIF2 } \alpha & \text { Eukaryotic initiation factor 2 } \alpha \\ \text { ER } & \text { Endoplasmic reticulum } \\ \text { GFP } & \text { Green fluorescent protein } \\ \text { GRP78 } & \text { Glucose-regulated protein } \\ \text { HOG-LDL } & \text { Highly oxidised glycated LDL }\end{array}$




$\begin{array}{ll}\text { HRCP } & \text { Human retinal capillary pericyte } \\ \text { JNK } & \text { c-Jun NH(2)-terminal kinase } \\ \text { N-LDL } & \text { Native LDL } \\ \text { PARP } & \text { Poly ADP ribose polymerase } \\ \text { PERK } & \begin{array}{l}\text { Protein kinase R-like endoplasmic } \\ \text { reticulum kinase }\end{array} \\ \text { RPE } & \text { Retinal pigment epithelium } \\ \text { ROS } & \text { Reactive oxygen species } \\ \text { siRNA } & \text { Small interfering RNA } \\ \text { STZ } & \text { Streptozotocin } \\ \text { UPR } & \text { Unfolded protein response } \\ \text { VEGF } & \text { Vascular endothelial growth factor } \\ \text { WT } & \text { Wild-type }\end{array}$

\section{Introduction}

Diabetic retinopathy remains a major cause of visual impairment in the working-age population [1]. Pericytes are critical in maintaining retinal vascular integrity [2]; their loss is considered an initiating event of diabetic retinopathy [3-6] but the mechanisms of pericyte loss are poorly understood, hindering development of effective therapies.

Autophagy, a catabolic process by which cells degrade and recycle their own constituents through a lysosomal mechanism, acts as a cellular sensor of intra- and extracellular stresses $[7,8]$. In diabetes, misfolded proteins accumulate in the endoplasmic reticulum (ER) leading to ER stress. ER stress activates the unfolded protein response (UPR), restoring protein homeostasis and promoting cell survival. Autophagy is an alternative pathway to the UPR. Rapamycin, an inducer of autophagy, inhibits angiogenic sprouting and vascular endothelial growth factor (VEGF) production in a co-culture model of retinal pigment epithelial (RPE) and endothelial cells [9-11], and in diabetic rats it suppresses retinal oxidative stress and VEGF expression [10] and prevents age-related retinopathy [11]. Targeting autophagy may therefore have therapeutic potential; however, in certain circumstances, autophagy may activate apoptotic death [12], and depending on context, stress-induced autophagy may promote survival or death of a given cell species [13].

Diabetic retinopathy is generally viewed as a consequence of hyperglycaemia, but in previous studies we showed that extravasated modified LDL also plays a critical role [14-24]. The effects of extravasated lipoproteins in atherosclerosis are well-established [25]; we have demonstrated analogous effects in the retina once the blood-retinal barrier (BRB) integrity is compromised, or bypassed, as in an animal model we described recently [26]. Initial leakage may be mild and transient but, as extravasated LDL accumulates, vicious cycles of damage may be established. While oxidised lipoproteininduced autophagy is implicated in atherogenesis [27], little is known regarding the retina. Previously, we showed that in human retinal capillary pericytes (HRCPs) and retinal pigment epithelium (RPE), ER stress that had been induced by highly oxidised glycated human LDL is mitigated by the UPR, but that in the presence of continued severe stresses ER homeostasis could not be preserved, resulting in apoptosis $[22,23]$. In this study, we aim to determine the underlying mechanisms whereby autophagy and apoptosis contribute to pericyte death.

\section{Methods}

Ethics The study was approved by the Institutional Review Board at the University of Oklahoma Health Sciences Centre (OUHSC) and the Ethics Committee at the Queen's University of Belfast, and was conducted according to the principles of the Declaration of Helsinki. Animal experiments were approved by the Institutional Animal Care and Use Committee at the Medical University of South Carolina and by the Queen's University Belfast Ethical Review Committee for Animal Research. All the animal experiments were randomised and blind to group assignment and outcome assessment.

Immunohistochemistry of human retinas Human retinas were obtained post-mortem from the National Disease Research Interchange (NDRI; Philadelphia, PA, USA) as described [23, 24]. Retinas were from age-matched individuals categorised as follows: non-diabetic, diabetic without clinical diabetic retinopathy; diabetic with retinopathy ( $n=3$ or 4 per group). The antibody used for immuno-histochemical detection of autophagy marker LC3B (catalogue No. 3868, 1:100 dilution; Cell Signaling Technology, Danvers, MA, USA) was also used in western blots (below): according to the manufacturer, it recognises human and murine LC3BI and LC3BII, and may exhibit some cross-reactivity with LC3A. Absence of non-specific tissue binding by secondary antibodies was confirmed. See Methods in electronic supplementary material (ESM) for details of retinal sample preparation and immunohistochemistry.

LDL preparation, modification and characterisation Lipoproteins were prepared as described [23, 28]. Briefly, native LDL (N-LDL) was prepared by sequential ultracentrifugation of freshly pooled plasma from healthy human volunteers. Highly oxidised glycated LDL (HOG-LDL) was prepared by glycating N-LDL, then oxidising with $\mathrm{CuCl}_{2}$. See ESM Methods for details.

Genetically modified mouse model of hyperlipidaemia Genetically modified C57B16 male mice (Genentech, South San Francisco, CA, USA) with double knockout of the genes encoding the LDL receptor $\left(\mathrm{Ldlr}^{-/-}\right)$and apolipoprotein B 
mRNA-editing catalytic polypeptide (converts ApoB100 to ApoB48) $\left(\right.$ Apobec $^{-1-}$ ) were used to model hypercholesterolaemia (vs wild-type [WT] controls) [29]. When mice reached 7 weeks of age, diabetes was induced by streptozotocin (STZ) as described [30], yielding groups with and without diabetes and with and without hypercholesterolaemia. All mice were maintained under a $12 \mathrm{~h}$ light-12 h dark cycle (07:00-19:00 hours) and constant temperature $\left(25^{\circ} \mathrm{C}\right)$ throughout the study, with food and water given ad libitum. Retinas were studied 40 weeks after diabetes induction: see ESM Methods for details.

Diabetes induction and LDL intravitreal injection in rats Diabetes was induced by STZ in adult (8-10 weeks) male Sprague-Dawley rats weighing 280-330 g. After 8 weeks, human HOG-LDL or N-LDL $(5 \mu \mathrm{l}, 0.5$ or $2.0 \mathrm{~g}$ protein/ 1 in PBS, to yield 50 or $200 \mathrm{mg} / 1$ final intravitreal concentration), or PBS alone, was injected intravitreally. After 7 days, retinas were harvested for western blot. The rats were maintained under a $12 \mathrm{~h}$ light-12 h dark cycle (07:00-19:00 hours) and constant temperature $\left(25^{\circ} \mathrm{C}\right)$ throughout the study, with food and water given ad libitum. See ESM Methods for details.

HRCP cell culture HRCPs (Cambrex, Walkersville, MD, USA) were cultured in EBM-2 medium. Cells (passages 3-9) at 85\% confluence were treated with either N-LDL or HOG-LDL as indicated. Where appropriate, cells were pre-treated with pharmacological reagents prior to lipoprotein exposure. See ESM Methods for details.

Cell viability assay HRCPs were studied in 96-well plates $\left(1 \times 10^{4}\right.$ cells/well $)$. Cell viability was measured using a cell counting assay (CCK-8; Dojindo Molecular Technologies, Rockville, MD, USA), per the manufacturer's protocol.

Western blotting Human retinas or pericytes were homogenised with a complete lysis buffer (Roche, Indianapolis, IN, USA). Protein concentrations were determined by the BCA protein assay (Pierce, Rockford, IL, USA). Protein (30 $\mu \mathrm{g}$ ) was resolved by SDS-PAGE and then blotted with antibodies against autophagyrelated homologue 5 (ATG-5), Beclin-1, LC3B (detects both LC3B-I and LC3B-II; some cross-reactivity with LC3A), phosphorylated c-Jun NH(2)-terminal kinase (p-JNK), total JNK, cleaved poly ADP ribose polymerase (PARP), activated caspase-3, C/EBP-homologous protein (CHOP), $\beta$-actin (1:3000) (all from Cell Signaling Technology); and phosphorylated-protein kinase R-like ER kinase (p-PERK), $78 \mathrm{kDa}$ glucose-regulated protein (GRP78) and phosphorylated eukaryotic initiation factor $2 \alpha$ (eIF2 $\alpha$ ) (all from Abcam, Cambridge, MA, USA). All antibodies were diluted 1:1000 unless indicated otherwise. Antibody to $\beta$-actin was used as a loading control. All cell culture experiments were repeated three times independently and quantified by densitometry.
TUNEL assay for apoptosis HRCPs were seeded and grown to $85 \%$ confluence on glass coverslips, and treated as indicated. Apoptosis was assessed (in situ cell death detection kit; Roche, Indianapolis, IN, USA) as per manufacturer's instructions. Immunofluorescence was visualised under a fluorescence microscope (Nikon, Tokyo, Japan).

Measurement of intracellular reactive oxygen species Reactive oxygen species (ROS) were measured with chloromethyl derivative of H2DCFDA (CM-H2DCFDA) (Life Technologies, Invitrogen, Carlsbad, CA, USA) as previously described [24]. Briefly, cells were seeded in 96-well plates $\left(1 \times 10^{4}\right.$ cells/well $)$. When they reached $80 \%$ confluence, cells were washed and incubated with $20 \mu \mathrm{mol} / 1$ DCFDA at $37^{\circ} \mathrm{C}$ for $20 \mathrm{~min}$, then exposed to experimental conditions. Fluorescence was measured at an excitation wavelength of $495 \mathrm{~nm}$ and an emission wavelength of $525 \mathrm{~nm}$ (VICTOR3 microplate reader; PerkinElmer, Waltham, MA, USA).

Data analyses Data are expressed as means \pm SD. Statistical significance was determined by Student's $t$ test or one-way ANOVA followed by post hoc Dunnett's test as appropriate (Prism 5 software; Graphpad, La Jolla, CA, USA). A $p$ value of $\leq 0.05$ was considered significant.

\section{Results}

Autophagy in human diabetic retina LC3B immunohistochemistry was performed in retinas from individuals with type 2 diabetes with and without diabetic retinopathy, and from non-diabetic individuals. In diabetic retinas, punctate staining (indicating autophagosomes) was observed in the ganglion cell layer and inner nuclear layer, but in non-diabetic retinas, punctate staining was absent (Fig. 1a). Retinal protein lysates were analysed (western blotting) for LC3B and two other autophagy markers, ATG-5 and Beclin-1. LC3B and ATG-5 were higher in diabetic vs non-diabetic individuals, but retinopathy status had no effect; Beclin-1 levels tended to be higher in diabetic retinas (Fig. 1b). Overall, autophagy was increased in the diabetic retina; the similarity between those with and without retinopathy may reflect pre-clinical injury in people who appear disease-free.

Autophagy in diabetic and hypercholesterolaemic mouse retina $L C 3 B$ staining was significantly higher in the two diabetic groups vs the non-diabetic group, with hyperlipidaemic diabetic mice showing the greatest intensity, localised predominantly in the ganglion cell and inner nuclear layers (ESM Fig. 1). Again, retinal autophagy was increased in the presence of diabetes, and more so in the added presence of long-standing hypercholesterolaemia. 
Fig. 1 Autophagy is evident in diabetic human retinas. (a) Immunohistochemistry for LC3B in human retinal sections: nondiabetic (non-DM), diabetic without clinical retinopathy (DM) and diabetic with clinical retinopathy (DR). DAPI (blue) was used to visualise the nuclei. Scale bar, $20 \mu \mathrm{m}$. GCL, ganglion cell layer; INL, inner nuclear layer; ONL, outer nuclear layer. Punctate staining of LC3B (green) was present in both groups of diabetic retinas but was minimal in non-diabetic retinas.

There was no obvious difference between the two diabetic groups. (b) Western blots for ATG-5 (white bars), Beclin-1 (grey bars) and LC3B (black bars) were performed on total retinal protein extracts from individual human retinas and quantified by densitometry (mean $\pm \mathrm{SD}, n=3$ or $4, * p<0.05$ vs non-DM)
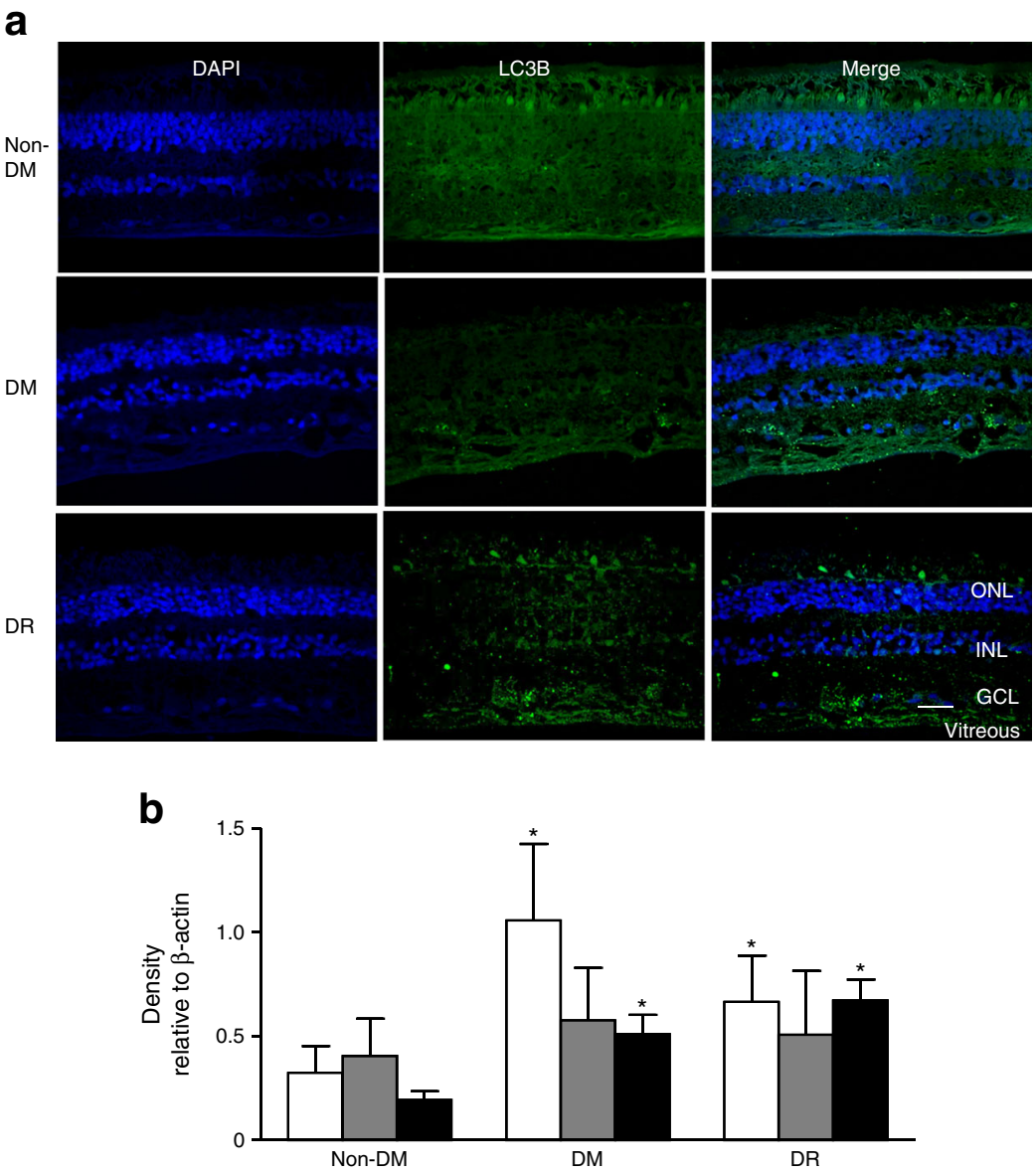

HOG-LDL induces autophagy in pericytes To determine whether modified LDL elicits autophagy in vitro, cultured HRCPs were transfected with a green fluorescent protein (GFP)-labelled LC3 plasmid, then exposed to HOG-LDL vs NLDL. HOG-LDL elicited a punctate intracellular GFP-LC3B distribution, characteristic of autophagy [31], which was not observed in response to N-LDL (Fig. 2a and ESM Fig. 2). The effect was further enhanced by chloroquine (CQ), an autophagosomelysosome fusion blocker [32], confirming that HOG-LDL enhances autophagic flux. In concert, western blots of HRCP lysates showed increased levels of LC3BII (the lipidated form present in autophagosomes) in response to HOG-LDL vs N-LDL, and a further increase in response to HOG-LDL + CQ (Fig. 2b). HOG-LDL increased protein expression of ATG-5, Beclin-1 and LC3BII in a dose-dependent manner over the concentration range $0-50 \mathrm{mg} / 1$, but caused no further increase at concentrations from 50 to $200 \mathrm{mg} / \mathrm{L}$ (Fig. 2c). Concentrations $>200 \mathrm{mg} / \mathrm{l}$ were not tested due to cellular toxicity.

JNK mediates HOG-LDL-induced ER stress and autophagy Jun amino-terminal kinases have been implicated in stress-induced autophagy [33, 34]. In cultured HRCPs, HOGLDL vs N-LDL significantly increased JNK phosphorylation
(Fig. 2d), to an extent equivalent to that induced by tunicamycin, an ER stress inducer. This response was obliterated by pre-treatment with sodium phenylbutyrate, an ER stress inhibitor, confirming that JNK mediates HOG-LDLinduced ER stress in pericytes.

To determine the role of JNK in autophagy, JNK phosphorylation inhibitor SP60012 was employed. JNK pathway inhibition was confirmed by western blot (ESM Fig. 3a). SP60012 pre-treatment attenuated HOG-LDL-induced autophagy, as demonstrated by decreased expression of LC3BII, ATG-5 and Beclin-1 (Fig. 2e). JNK knockdown using small interfering RNA (siRNA) had a similar effect, reducing both phosphorylated and total JNK (ESM Fig. 3b) as well as HOG-LDL-induced autophagy (Fig. 2f). The data support an essential role for JNK activation in the mediation of HOG-LDL-induced autophagy in HRCPs.

A dual role for autophagy in HOG-LDL-induced pericyte death HOG-LDL caused dose-dependent toxicity to cultured HRCPs: no death occurred up to $50 \mathrm{mg} / \mathrm{l}$, but viability decreased progressively from $50 \mathrm{mg} / 1$ to $300 \mathrm{mg} / \mathrm{l}$ (Fig. 3a). To understand the relative involvement of autophagy and apoptosis, we employed 3-methyladenine (3-MA), a specific 


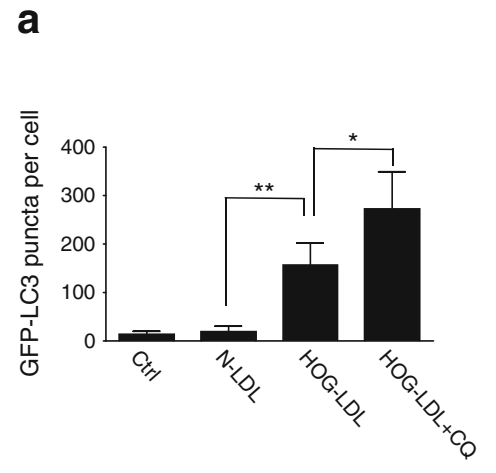

b

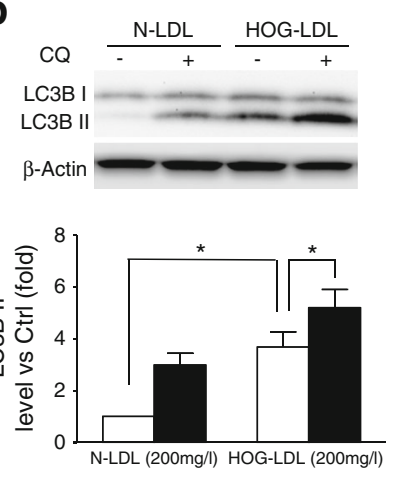

C

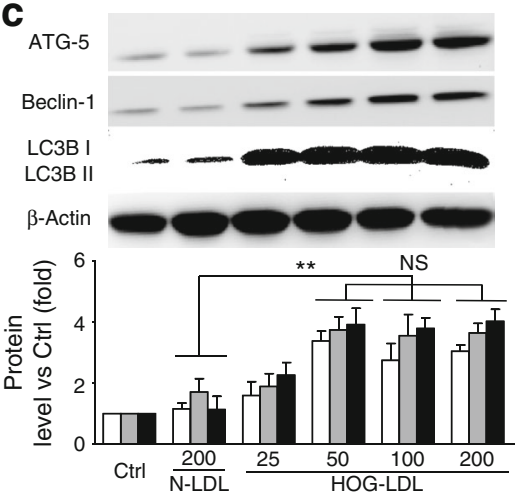

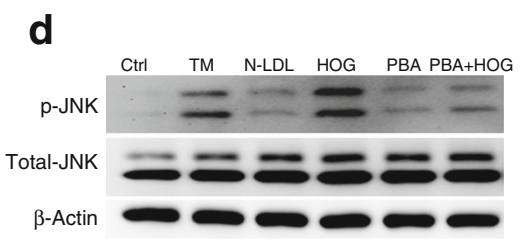

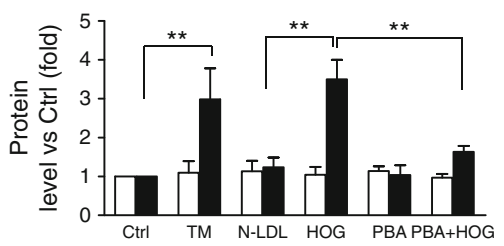

e
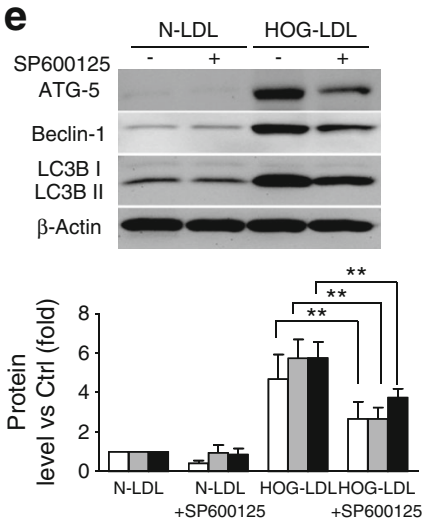

f
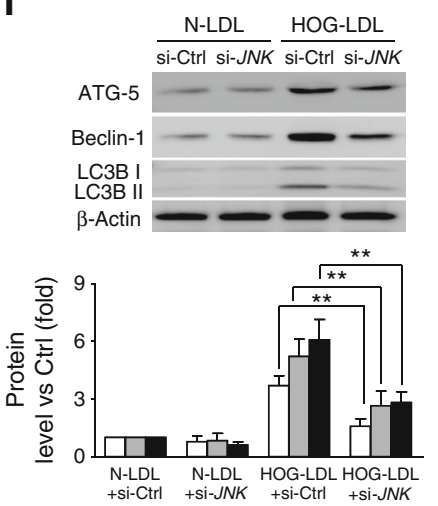

Fig. 2 HOG-LDL induces JNK phosphorylation-dependent autophagy in HRCPs. (a) HRCPs were transfected with GFP-LC3B plasmid for $36 \mathrm{~h}$, then exposed to N-LDL (200 mg/l) or HOG-LDL (200 mg/l) for $24 \mathrm{~h}$, with or without CQ $(10 \mu \mathrm{mol} / \mathrm{l})$ pre-treatment for $1 \mathrm{~h}$. Untreated cells served as control (Ctrl) in this and the following figs. Autophagy was quantified by counting GFP-LC3B puncta within cells ( $n=5$ experiments; ${ }^{*} p<0.05,{ }^{*} p<0.01$ ). (b) HRCPs were directly exposed to LDL with or without CQ and analysed by western blot for LC3BII. White bars, control; black bars, CQ (mean $\pm \mathrm{SD}, n=3 ;{ }^{*} p<0.05$ ). (c) HRCPs were exposed to N-LDL (200 mg/l) or HOG-LDL (25-200 mg/l) for $12 \mathrm{~h}$ and analysed by western blot. ATG-5 (white bars), Beclin-1 (grey bars) and LC3BII (black bars) levels were quantified (mean $\pm \mathrm{SD}, n=3$;
$* * p<0.01)$. (d) HRCPs were treated with tunicamycin (TM; $2 \mu \mathrm{mol} / \mathrm{l})$, N-LDL $(200 \mathrm{mg} / \mathrm{l})$ or HOG-LDL $(200 \mathrm{mg} / \mathrm{l})$ for $12 \mathrm{~h}$ or pre-treated with 4-phenylbutyric acid (PBA; $10 \mathrm{mg} / \mathrm{l}$ ) before exposure to HOG-LDL. Western blot analysis was carried out and total JNK (white bars) and p-JNK (black bars) levels were quantified (mean $\pm \mathrm{SD}, n=3$; $\left.{ }^{* *} p<0.01\right)$. (e, f) HRCPs were pre-treated with p-JNK inhibitor SP600125 $(10 \mu \mathrm{mol} / \mathrm{l})$ for $1 \mathrm{~h}(\mathbf{e})$, or transfected with siRNA against JNK (si-JNK) or with si-Ctrl for $36 \mathrm{~h}(\mathbf{f})$, then exposed to N-LDL (200 mg/l) or HOG-LDL (200 mg/l) for $12 \mathrm{~h}$. Western blotting was carried out and levels of ATG-5 (white bars), Beclin-1 (grey bars) and LC3BII (black bars) were quantified (mean $\pm \mathrm{SD}, n=3$; ** $p<0.01$ ) inhibitor of phosphoinositide 3-kinase and the initial phase of autophagy, and Z-VAD-fmk, a caspase inhibitor. As shown in Fig. 3b, at a non-toxic HOG-LDL concentration of $50 \mathrm{mg} / \mathrm{l}$, 3-MA triggered pericyte death, which was prevented by the apoptosis inhibitor Z-VAD. This suggests a pro-survival role for autophagy under mild, sub-lethal stress $(50 \mathrm{mg} / \mathrm{l} \mathrm{HOG-}$ LDL). However, at a higher HOG-LDL concentration $(200 \mathrm{mg} / \mathrm{l}), 3-\mathrm{MA}$ attenuated cell death, adding to the rescuing effect of Z-VAD (Fig. 3b). As 3-MA may not provide specific inhibition of autophagy, siRNA against Beclin-1 was also used: Beclin-1 knockdown decreased pericyte viability after exposure to $50 \mathrm{mg} / 1$ HOG-LDL but enhanced it after exposure to $200 \mathrm{mg} / \mathrm{l} \mathrm{HOG-LDL} \mathrm{(Fig.} \mathrm{3c).} \mathrm{The} \mathrm{data} \mathrm{suggest} \mathrm{that} \mathrm{under}$ severe cellular stresses, autophagy shifts from a protective to an injurious role. This observation was further supported by western blotting analysis and TUNEL assay (Fig. 3d-f).
HOG-LDL induced apoptosis at concentrations of 100 and $200 \mathrm{mg} / \mathrm{l}$, demonstrated by increased levels of cleaved PARP, activated caspase 3 and TUNEL-positive apoptotic cells at $200 \mathrm{mg} / \mathrm{l}$ but not at $50 \mathrm{mg} / \mathrm{l}$. However, inhibition of autophagy by 3-MA and CQ induced apoptosis at a HOGLDL concentration of $50 \mathrm{mg} / \mathrm{l}$ but had no effect at $200 \mathrm{mg} / \mathrm{l}$, indicating the protective role of autophagy at mild but not at severe levels of HOG-LDL-induced stress (Fig. 3d-f and ESM Fig. 4a).

Comparison of the dose-response relationships for HOGLDL-induced oxidative stress, ER stress, JNK activation and CHOP expression To elucidate underlying signalling pathways and mechanisms, we compared dose-response relationships of oxidative stress (ROS), ER stress (chaperone: GRP78; sensors: p-PERK, p-eIF2 $\alpha$ ), JNK activation and 


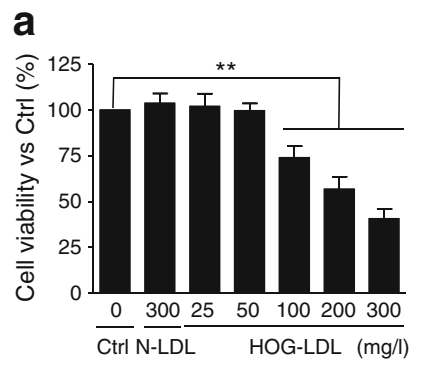

b

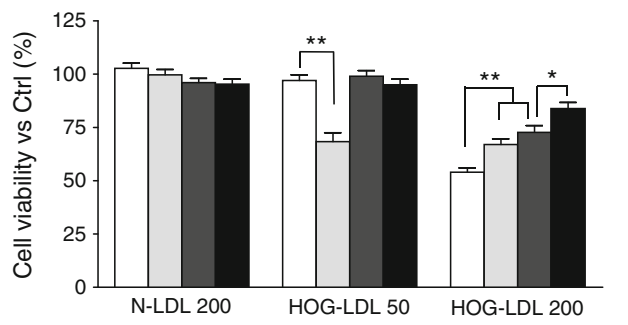

e
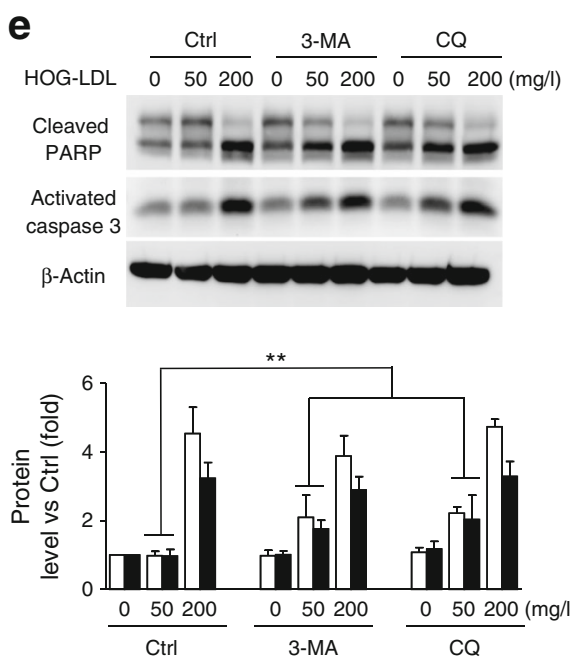

**
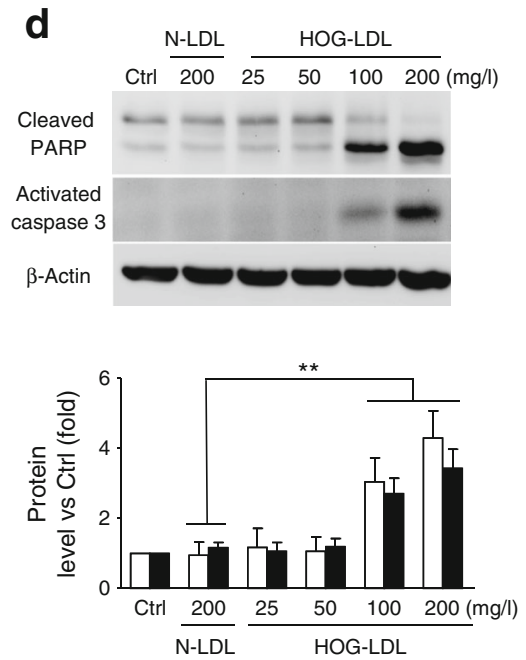

$\mathrm{N}$-LDL HOG-LDL

Fig. 3 A dual role for autophagy in HOG-LDL-induced pericyte death. (a-c) HRCPs were treated as follows: exposure to N-LDL (300 mg/l) or HOG-LDL (25-300 mg/l) for $24 \mathrm{~h}$ (a); pre-treatment with 3-MA ( $5 \mathrm{mmol} / \mathrm{l}$, light grey bars), Z-VAD-fmk $(100 \mu \mathrm{mol} / \mathrm{l}$, dark grey bars) and both of them (black bars) for $1 \mathrm{~h}(\mathbf{b})$; transfection with siRNA against Beclin-1 (si-Beclin-1, black bars) or si-Ctrl (white bars) for $36 \mathrm{~h}$, then exposure to HOG-LDL ( $50 \mathrm{mg} / 1,200 \mathrm{mg} / \mathrm{l})$ for $12 \mathrm{~h}(\mathbf{c})$. Cell viability was expressed as percentage vs control. (d-f) HRCPs: apoptosis and autophagy. Apoptosis is triggered by HOG-LDL at 100 or $200 \mathrm{mg} / \mathrm{l}$ : cleaved

CHOP expression in cultured HRCPs exposed to HOG-LDL. HOG-LDL increased levels of ROS, GRP78, p-PERK and peIF2 $\alpha$ dose-dependently (25-200 mg/l) (Fig. 4a, b). JNK activation increased over the $25-50 \mathrm{mg} / \mathrm{l}$ HOG-LDL range, then remained constant up to $200 \mathrm{mg} / \mathrm{l}$ (Fig. 4c), similar to autophagy (Fig. 2c). HOG-LDL did not increase CHOP expression until its concentration reached $100 \mathrm{mg} / \mathrm{l}$ (Fig. 4d), consistent with effects on apoptosis (Fig. 3d). Together, the data suggest that low-dose HOG-LDL (up to $50 \mathrm{mg} / \mathrm{ml}$ ) induces mild oxidative and ER stress, triggering a protective action of autophagy via JNK; at higher concentrations (100-200 mg/l), HOG-LDL further increases stresses leading to autophagic and apoptotic death.

CHOP, not $\mathrm{JNK}$, is responsible for HOG-LDL-induced apoptosis Both CHOP and JNK have been implicated in ER stress-induced apoptosis [35]. To determine their relative roles in HOG-LDL-induced apoptosis, we employed siRNA against $C H O P($ si-CHOP) or $J N K$ (si-JNK), then measured apoptosis (TUNEL assay, western blots). si-CHOP significantly reduced TUNEL-positive staining (Fig. 5a, ESM Ctrl, control
C

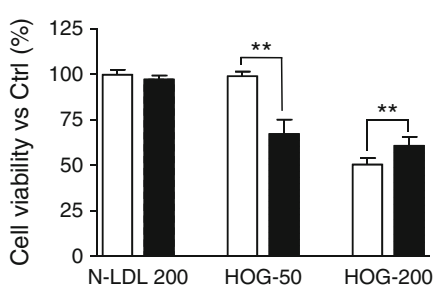

f

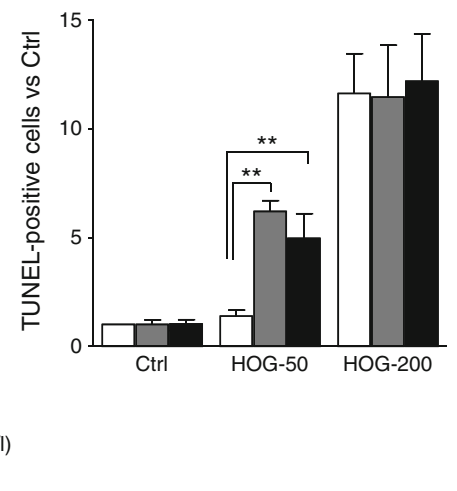

PARP (white bars), activated caspase-3 (black bars) were detected by western blot (d). HRCPs were pre-treated with 3 -MA $(5 \mathrm{mmol} / \mathrm{l})$ or CQ (10 $\mu \mathrm{mol} / \mathrm{l})$ for $1 \mathrm{~h}$ : cleaved PARP (white bars) and activated caspase-3 (black bars) were detected by western blot (e). HRCPs were pre-treated with vehicle (white bars) or 3-MA (5 mmol/l, grey bars) for $1 \mathrm{~h}$, or were transfected with si-Beclin-1 (black bars), then exposed to HOG-LDL $(50 \mathrm{mg} / \mathrm{l}, 200 \mathrm{mg} / \mathrm{l})$ for $12 \mathrm{~h}$ : TUNEL staining was expressed as a ratio of control (f). All data are means $\pm \mathrm{SD}, n=3$ or $5 ;{ }^{*} p<0.05,{ }^{*} p<0.01$.

Fig. 4b), cleaved PARP and activated caspase-3 (Fig. 5b), indicating a role for $\mathrm{CHOP}$ in apoptosis. However, in pericytes exposed to $50 \mathrm{mg} / \mathrm{l}$ HOG-LDL, si- $J N K$ increased protein levels of CHOP, cleaved PARP and activated caspase-3 (Fig. 5c), responses that promote apoptosis. In contrast, in the presence of $200 \mathrm{mg} / 1$ HOG-LDL, si-JNK had no effect. This is consistent with JNK knockdown inhibiting autophagy, thus blocking the protective effects of autophagy at lower levels of cell stress. Finally, we showed that si-CHOP did not change expression of p-JNK or LC3BII in pericytes exposed to HOG-LDL at 50 or $200 \mathrm{mg} / 1$ (Fig. 5 d), indicating that autophagy induced by HOG-LDL, in contrast to apoptosis, was CHOP-independent.

Extravascular HOG-LDL dose-dependently induced ER stress, autophagy and apoptosis in diabetic rat retinas To define responses in vivo, we evaluated retinas of diabetic rats, in which human N-LDL or HOG-LDL had been injected 7 days previously into the vitreous, to simulate chronic exposure to extravasated, modified LDL in human diabetic 
a

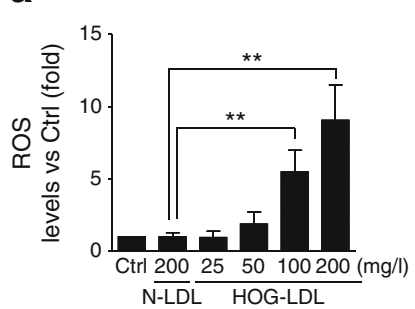

C

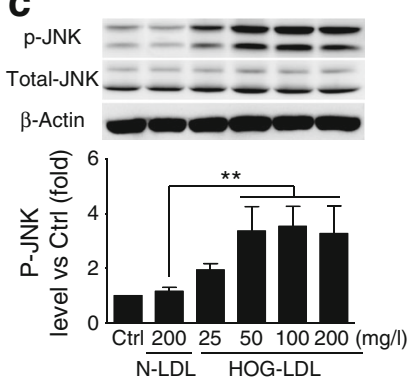

b

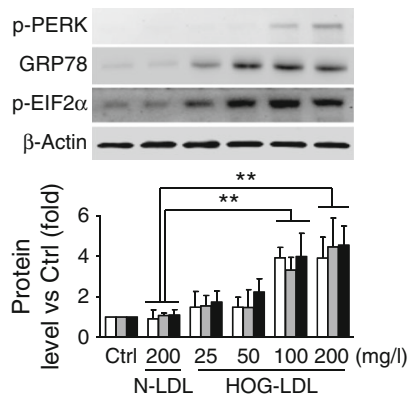

d

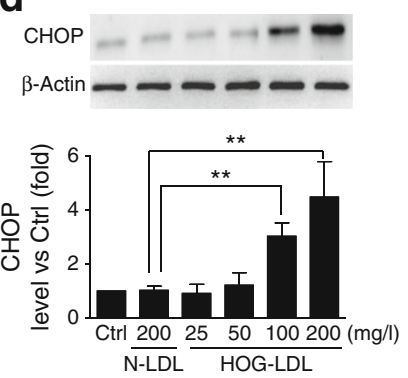

Fig. 4 HOG-LDL dose-responses for ROS generation, ER stress, JNK activation and CHOP expression. Cellular ROS level are shown following exposure to various doses of HOG-LDL (mean $\pm \mathrm{SD}, n=3$; $* * p<0.01$ vs N-LDL) (a). Western blots are shown for p-PERK (white bars), GRP78 (grey bars) and p-eIF2 $\alpha$ (black bars) (b), p-JNK and total JNK (c), and CHOP (d). Data are means $\pm \mathrm{SD}, n=3 ;{ }^{* *} p<0.01$. Ctrl, control

Fig. 5 CHOP, but not JNK, is essential for HOG-LDL-induced apoptosis. HRCPs were transfected with si-CHOP or si- $J N K$ for $36 \mathrm{~h}$, then exposed to N-LDL (N; $200 \mathrm{mg} / \mathrm{l})$ or HOGLDL (HOG; 50, $200 \mathrm{mg} / \mathrm{l}$ ) for 12 h. At $200 \mathrm{mg} / \mathrm{l} \mathrm{HOG-LDL}$, si-CHOP decreased TUNELpositive cells (a). At $200 \mathrm{mg} / \mathrm{l}$, si-CHOP decreased HOG-LDLinduced expression of CHOP (white bars), cleaved PARP (grey bars) and activated caspase- 3 (black bars) (b). At 50 mg/l HOGLDL, si-JNK increased expression of CHOP (white bars), cleaved PARP (grey bars) and activated caspase-3 (black bars) but had no effect at $200 \mathrm{mg} / \mathrm{l}$ (c). si-CHOP did not alter expression of p-JNK (white bars) or LC3BII (black bars) at 50 or $200 \mathrm{mg} / 1$ HOG-LDL (d). Data are means $\pm \mathrm{SD}, n=3 ; * p<0.05,{ }^{*} p<<0.01$ retinopathy, as recently described in a mouse model [26]. Two intravitreal concentrations, 50 and $200 \mathrm{mg} / \mathrm{l}$, were used to induce different degrees of retinal stress. Intravitreal HOG-LDL, but not N-LDL, induced ER stress, autophagy and apoptosis (Fig. 6). ER stress sensors (p-PERK, p-eIF2a) increased in a dose-dependent manner (Fig. 6a), whereas p-JNK and autophagy markers were increased to a similar extent at both doses (Fig. 6b, c). Apoptosis (CHOP, activated caspase 3) was observed only at $200 \mathrm{mg} / \mathrm{l} \mathrm{HOG-LDL} \mathrm{(Fig.} \mathrm{6d).} \mathrm{These} \mathrm{findings}$ are in agreement with the cell culture studies.

\section{Discussion}

Therapies targeting autophagy are of increasing interest and may be effective in retinal disease [9-11] but little is known regarding diabetic retinopathy. In the current study, we showed for the first time that autophagy markers (by immunohistochemistry and western blot) were increased in human retinas in the presence of diabetes, with or without concomitant retinopathy, and that in cell culture, exposure of pericytes to modified lipoproteins stimulated autophagy. These findings are consistent with our overall hypothesis that ectopic (extravasated) modified lipoproteins, when present in the diabetic retina, mediate responses (some defensive, some injurious) even before clinical diabetic retinopathy is evident a

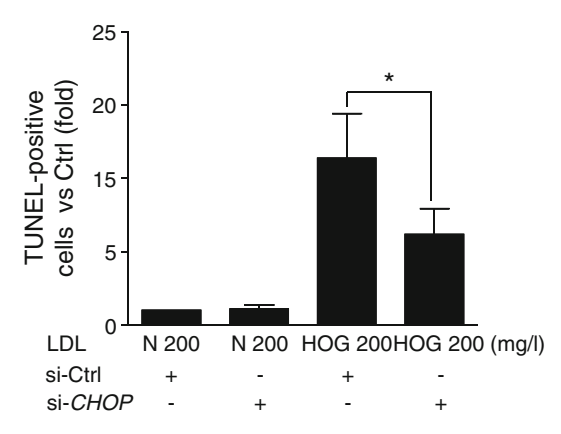

C

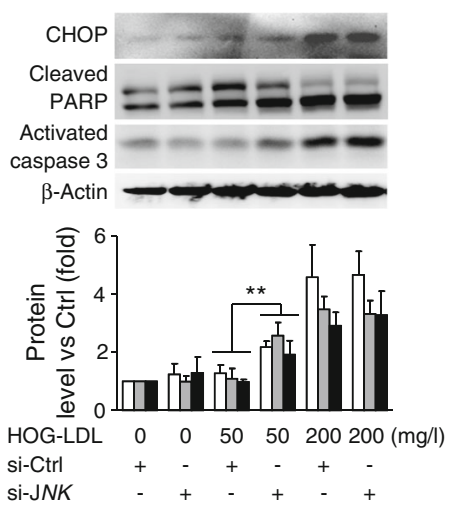

b

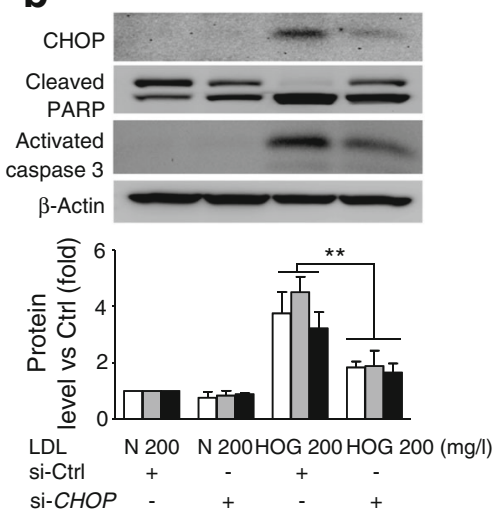

d

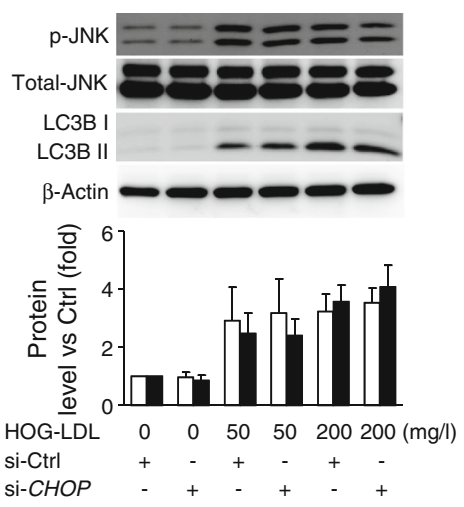


Fig. 6 Effects of intravitreal HOG-LDL on ER stress, JNK activation, autophagy and apoptosis in retinas of diabetic rats. STZ-induced diabetic rats received intravitreal HOG-LDL (HOG) vs N-LDL (N) to achieve vitreal levels of 50 or $200 \mathrm{mg}$ protein/l vs PBS control. Total retinal protein extracts were analysed by western blot, and the following protein levels were quantified by densitometry: p-PERK (white bars) and p-eIF $2 \alpha$ (grey bars) (a); p-JNK and total JNK (b); ATG-5 (white bars), Beclin-1 (grey bars) and LC3BII (black bars) (c); CHOP (white bars) and activated caspase 3 (grey bars) (d). Data are means $\pm \mathrm{SD}, n=5 ;{ }^{*} p<0.05$ and ${ }^{* *} p<0.01$ vs PBS; ${ }^{\dagger} p<0.05$ vs HOG-50
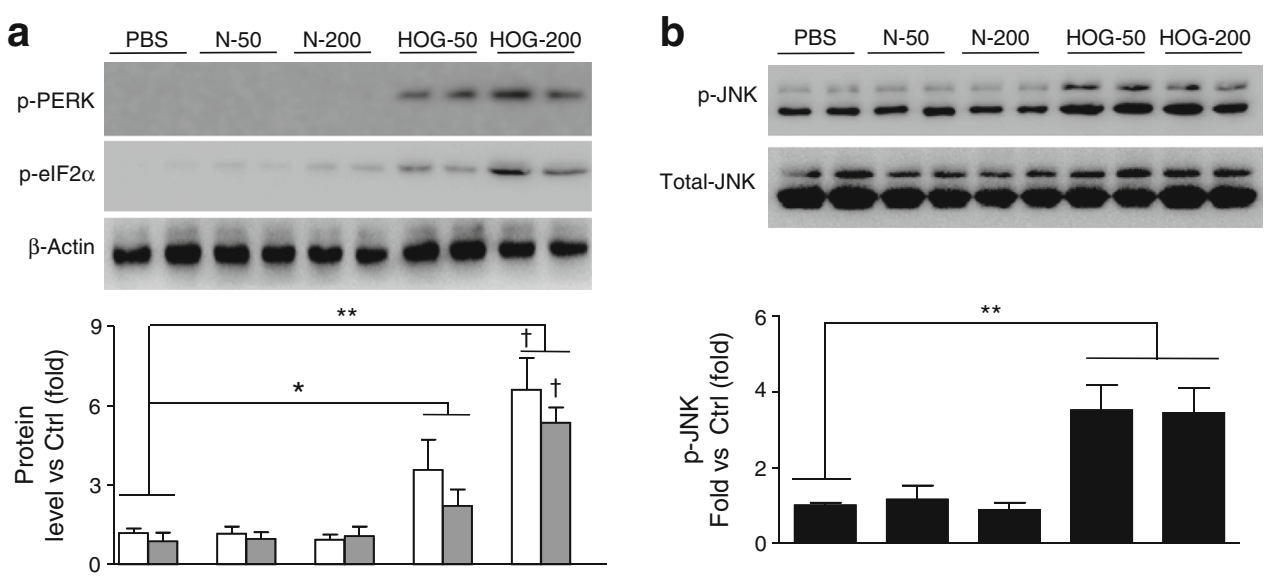

C
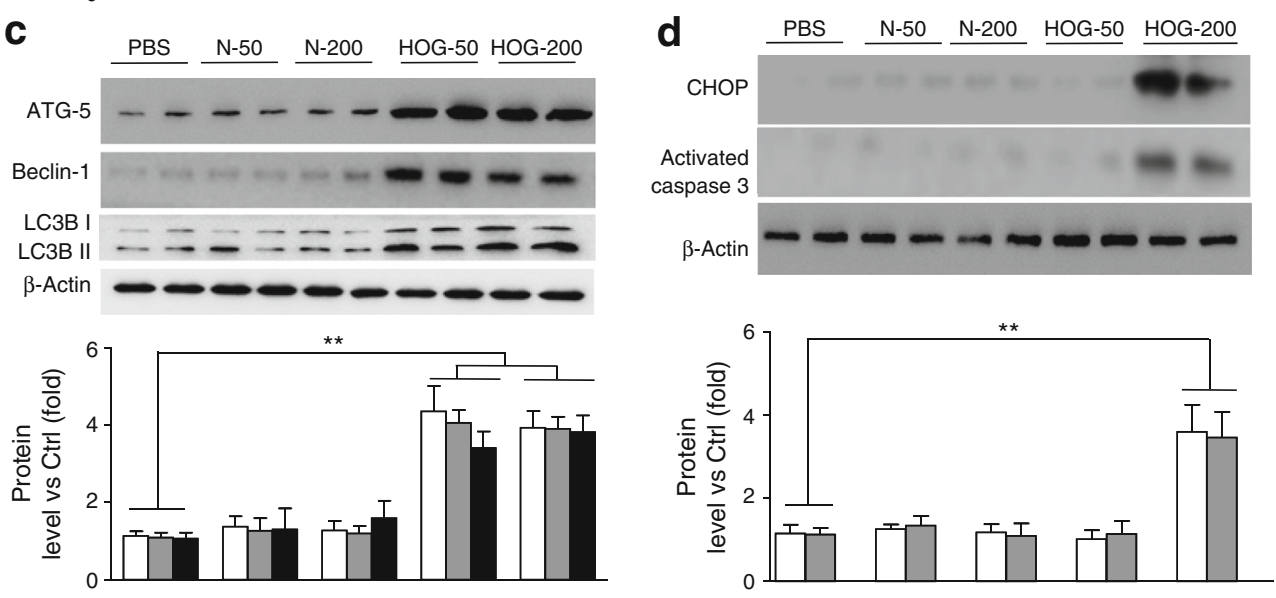

$[21,23]$. More interesting, our in vitro and in vivo data both suggest that autophagy may play a dual role: protecting against cell death under moderate stress, but contributing to it under severe stress (Fig. 7).

To maintain normal cellular function, autophagy may be upregulated in response to environmental stress. In the retina, all cell types rely on one or more aspects of autophagy to maintain structure and/or function [36]. Retinal autophagy was first observed (in rats) by Remé et al in 1977 [37], occurring primarily in the outer retina, where later it was shown to exhibit circadian variation [38]. More recently, Piano et al demonstrated upregulation of autophagy in retinal rods as an early feature of diabetic retinopathy (i.e. after 4-12 weeks diabetes) in STZ-induced diabetic mice [39]. In general, cellular homeostasis relies on regulated interplay between basal and stress-induced autophagic pathways [40]. Previously we showed that both extravasated modified lipoproteins and markers of ER stress were present in human diabetic retinas in proportion to the severity of retinopathy [23]. In the present study, the retinal findings from humans and genetically hypercholesterolaemic mice, with and without diabetes, are generally consistent with the findings described above: intra-retinal formation of autophagosomes (punctate LC3 staining) was increased by diabetes and further increased (in mice) by prolonged hypercholesterolaemia. In a new diabetic rat model using intravitreal LDL injection, we found additional supportive evidence: exposure of the retina to HOG-LDL but not N-LDL stimulated an autophagic response. Taken together, the data are consistent with autophagy being implicated in lipoprotein-mediated retinal injury and, specifically, from the cell culture work, in pericyte injury in diabetic retinopathy.

From the present data, we propose that the interplay between autophagy and apoptosis is critical for pericyte survival. The balance between survival and death depends on the level of stress - minor stress may be countered by autophagy but severe stress leads to cell death. This concept is in concert with the findings of Piano et al regarding effects of early diabetic retinopathy on retinal neural cells [39]. In the present work, the survival-death balance is seen in the observed dose-dependent effects of HOG-LDL on pericyte oxidative stress, ER stress, apoptosis and autophagy, with consistent findings following intravitreal LDL injections in rats, summarised in Fig. 7. HOG-LDL induced various molecular responses that were dose-dependent over different concentration ranges. Oxidative stress and ER stress were dose-dependent up to $200 \mathrm{mg} / \mathrm{l}$, autophagy up to $50 \mathrm{mg} / \mathrm{l}$ and apoptosis from $50 \mathrm{mg} / 1$ to $200 \mathrm{mg} / \mathrm{l}$. When cells 
a

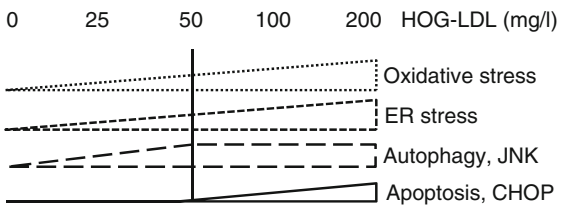

b

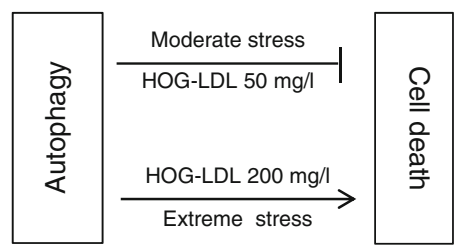

C

Working hypotheses

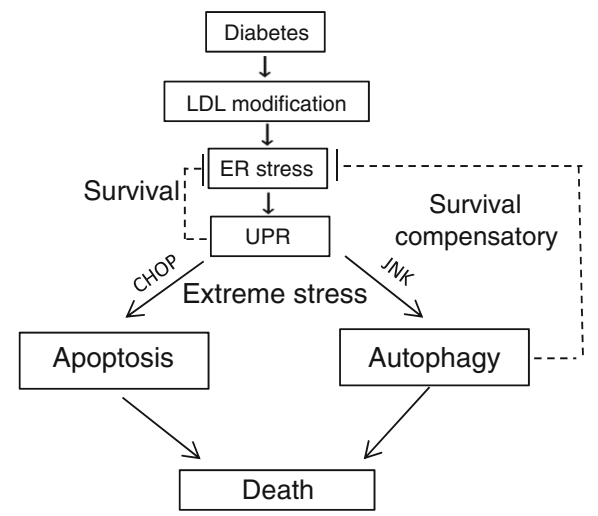

Fig. 7 Dual role for autophagy in stress-induced pericyte loss in diabetic retinopathy. (a) Summary of dose-dependent effects of HOG-LDL on oxidative stress, ER stress, autophagy and apoptosis in HRCPs. (b) Contrasting roles of autophagy in moderate vs severe HOG-LDL-induced cell stress. (c) Study hypothesis: in diabetic retina, modified LDL-triggered ER stress may be overcome by UPR or autophagy (as a pro-survival, compensatory pathway) but if cellular stress becomes more severe, both UPR and autophagy may shift to promote apoptotic and autophagic cell death

experienced relatively mild stress $(<50 \mathrm{mg} / \mathrm{l})$, autophagy was induced, promoting cell survival, but under more severe stress (100 or $200 \mathrm{mg} / \mathrm{l}$ ), autophagy was no longer protective but instead contributed to disruption of cellular homeostasis and death. Thus, pericytes utilise autophagy as a cytoprotective mechanism unless, or until, a critical stress threshold is exceeded. At that point, neither the UPR nor autophagy can protect the cells; instead, apoptotic mechanisms are activated and autophagy shifts from a protective to a lethal role. The definition of $50 \mathrm{mg} / \mathrm{l}$ as the threshold for this shift is clearly inexact, but nevertheless relevant in the retina where LDL is normally excluded by the BRB: any degree of BRB leakage would likely lead to accumulation and transition through this value. The pathophysiological relevance of the concentrations of HOG-LDL $(0-200 \mathrm{mg} / \mathrm{l})$ used in this study has been described and justified previously [21,23], and we believe them to be relevant in vivo.
A dual role for autophagy has been proposed in other studies. In rheumatoid arthritis, autophagy in synovial fibroblasts may promote cell survival or death, depending on the level of stress [41]. In the liver, autophagy is essential for maintenance of hepatocyte mitochondria and control of oxidative stress, thus preventing carcinogenesis, but once hepatocarcinoma is established, autophagy can promote the disease [42]. In diabetic retinopathy, apparently contradictory roles for autophagy can inhibit or promote retinal vascular injury, depending on context (e.g. severity of retinopathy, extent of LDL leakage, extent of modification). In early retinopathy, when BRB leakage is mild, the retina is exposed only to small quantities of modified LDL [18] and autophagy may enhance cell survival. As retinopathy progresses and BRB leakage and lipoprotein leakage/modification become more severe, neither UPR nor autophagy can maintain ER homeostasis and autophagic death ensues. This dual action may complicate the development of treatments for diabetic retinopathy that aim to modulate autophagy.

The JNK pathway has been implicated in a range of cellular stress responses [7, 33, 43]. In this study, we demonstrated that JNK phosphorylation was essential to autophagy induced by HOG-LDL and ER stress. This is consistent with data implicating PERK-eIF2a and IRE1-JNK signalling pathways in autophagy [7, 44, 45]. Apart from autophagy, JNK activation is implicated in ER stress-induced apoptosis [46]. However, in the present study, we showed that JNK 'knockdown' did not affect HOG-LDL (200 mg/l)-induced apoptosis, and enabled lower concentrations of HOG-LDL $(50 \mathrm{mg} / \mathrm{l})$ to induce apoptosis. This suggests that JNK activation was not implicated in HOG-LDL-induced apoptosis, but at low levels of stress it may promote autophagy and thus protect cells against apoptosis. It remains unclear whether or how activation of JNK through ER stress affects upstream components of the autophagy pathway (e.g. mechanistic target of rapamycin; mTOR) [47]. Further studies regarding the detailed pathway of HOG-LDL-induced autophagy, including the relative roles of LC3B and LC3A, which may not be clearly distinguished in the present study, are needed: possibly, differential effects of the two isoforms could be important.

In conclusion, we present further evidence that autophagy is present in human diabetic retinas, and a role for modified lipoproteins is supported by in vivo findings in diabetic mouse and rat retina and by in vitro studies of HRCPs. We show that ER stress-mediated autophagy may play a dual role in pericyte loss induced by modified LDL. At low levels of exposure autophagy has a pro-survival effect, but as stresses become severe it promotes cell death. This dual function has implications for the development of any future autophagy-based therapies, which might only be applicable early in disease evolution when intra-retinal stresses remain mild. 
Acknowledgements The NDRI provided valued assistance in obtaining human retinal tissues. We thank M. Leyva and T. Friedel for expert technical and logistic support.

Funding This work was supported by the Oklahoma Center for the Advancement of Science and Technology (HR08-067), by a Research Grant from the American Diabetes Association (7-12-CT46), and by the National Institutes of Health (USA) COBRE Program of the National Center for Research Resources (P20 RR 024215).

Duality of interest The authors declare that there is no duality of interest associated with this manuscript.

Contribution statement $\quad$ DF, SY, MW, SMH, ARC and MD conducted experiments, researched data and wrote the manuscript. JYY and JC researched data and wrote the manuscript. TJL conceived and conducted the study, researched data and wrote the manuscript. TJL is the guarantor of this work and, as such, had full access to all the data in the study and takes responsibility for the integrity of the data and the accuracy of the data analysis. All the authors have approved publication.

Open Access This article is distributed under the terms of the Creative Commons Attribution 4.0 International License (http:// creativecommons.org/licenses/by/4.0/), which permits unrestricted use, distribution, and reproduction in any medium, provided you give appropriate credit to the original author(s) and the source, provide a link to the Creative Commons license, and indicate if changes were made.

\section{References}

1. Gardner TW, Antonetti DA (2007) A prize catch for diabetic retinopathy. Nat Med 13:131-132

2. Hammes HP (2005) Pericytes and the pathogenesis of diabetic retinopathy. Horm Metab Res 37(Suppl 1):39-43

3. Cogan DG, Toussaint D, Kuwabara T (1961) Retinal vascular patterns. IV. Diabetic retinopathy. Arch Ophthalmol 66:366-378

4. Kern TS, Engerman RL (1995) Vascular lesions in diabetes are distributed non-uniformly within the retina. Exp Eye Res 60:545-549

5. Mizutani M, Kern TS, Lorenzi M (1996) Accelerated death of retinal microvascular cells in human and experimental diabetic retinopathy. J Clin Invest 97:2883-2890

6. Hammes HP, Lin J, Renner O et al (2002) Pericytes and the pathogenesis of diabetic retinopathy. Diabetes 51:3107-3112

7. Ogata M, Hino S, Saito A et al (2006) Autophagy is activated for cell survival after endoplasmic reticulum stress. Mol Cell Biol 26:9220-9231

8. Ding WX, Ni HM, Gao W et al (2007) Differential effects of endoplasmic reticulum stress-induced autophagy on cell survival. J Biol Chem 282:4702-4710

9. Stahl A, Paschek L, Martin G et al (2008) Rapamycin reduces VEGF expression in retinal pigment epithelium (RPE) and inhibits RPE-induced sprouting angiogenesis in vitro. FEBS Lett 582:3097-3102

10. Ozdemir G, Kilinc M, Ergun Y, Sahin E (2014) Rapamycin inhibits oxidative and angiogenic mediators in diabetic retinopathy. Can $\mathrm{J}$ Ophthalmol 49:443-449

11. Kolosova NG, Muraleva NA, Zhdankina AA, Stefanova NA, Fursova AZ, Blagosklonny MV (2012) Prevention of age-related macular degeneration-like retinopathy by rapamycin in rats. Am $\mathrm{J}$ Pathol 181:472-477
12. Levine B, Yuan J (2005) Autophagy in cell death: an innocent convict? J Clin Invest 115:2679-2688

13. Codogno P, Meijer AJ (2005) Autophagy and signaling: their role in cell survival and cell death. Cell Death Differ 12(Suppl 2):1509-1518

14. Lyons TJ, Li W, Wells-Knecht MC, Jokl R (1994) Toxicity of mildly modified low-density lipoproteins to cultured retinal capillary endothelial cells and pericytes. Diabetes 43:1090-1095

15. Lyons TJ, Li W, Wojciechowski B, Wells-Knecht MC, Wells-Knecht KJ, Jenkins AJ (2000) Aminoguanidine and the effects of modified LDL on cultured retinal capillary cells. Invest Ophthalmol Vis Sci 41:1176-1180

16. Lyons TJ, Jenkins AJ, Zheng D et al (2004) Diabetic retinopathy and serum lipoprotein subclasses in the DCCT/EDIC cohort. Invest Ophthalmol Vis Sci 45:910-918

17. Song W, Barth JL, Yu Y et al (2005) Effects of oxidized and glycated LDL on gene expression in human retinal capillary pericytes. Invest Ophthalmol Vis Sci 46:2974-2982

18. Wu M, Chen Y, Wilson $\mathrm{K}$ et al (2008) Intraretinal leakage and oxidation of LDL in diabetic retinopathy. Invest Ophthalmol Vis Sci 49:2679-2685

19. Diffley JM, Wu M, Sohn M, Song W, Hammad SM, Lyons TJ (2009) Apoptosis induction by oxidized glycated LDL in human retinal capillary pericytes is independent of activation of MAPK signaling pathways. Mol Vis 15:135-145

20. Zhou T, Zhou KK, Lee K et al (2011) The role of lipid peroxidation products and oxidative stress in activation of the canonical wingless-type MMTV integration site (WNT) pathway in a rat model of diabetic retinopathy. Diabetologia 54:459-468

21. Wu M, Yang S, Elliott MH et al (2012) Oxidative and endoplasmic reticulum stresses mediate apoptosis induced by modified LDL in human retinal Muller cells. Invest Ophthalmol Vis Sci 53:4595-4604

22. Du M, Wu M, Fu D et al (2013) Effects of modified LDL and HDL on retinal pigment epithelial cells: a role in diabetic retinopathy? Diabetologia 56:2318-2328

23. Fu D, Wu M, Zhang J et al (2012) Mechanisms of modified LDL-induced pericyte loss and retinal injury in diabetic retinopathy. Diabetologia 55:3128-3140

24. Fu D, Yu JY, Wu M et al (2014) Immune complex formation in human diabetic retina enhances toxicity of oxidized LDL towards retinal capillary pericytes. J Lipid Res 55:860-869

25. Berliner JA, Navab M, Fogelman AM et al (1995) Atherosclerosis: basic mechanisms. Oxidation, inflammation, and genetics. Circulation 91:2488-2496

26. Yu JY, Du M, Elliott MH et al (2016) Extravascular modified lipoproteins: a role in the propagation of diabetic retinopathy in a mouse model of type 1 diabetes. Diabetologia doi:10.1007/s00125-0164012-6

27. Martinet W, De Meyer GR (2009) Autophagy in atherosclerosis: a cell survival and death phenomenon with therapeutic potential. Circ Res 104:304-317

28. Jenkins AJ, Velarde V, Klein RL et al (2000) Native and modified LDL activate extracellular signal-regulated kinases in mesangial cells. Diabetes 49:2160-2169

29. Powell-Braxton L, Veniant M, Latvala RD et al (1998) A mouse model of human familial hypercholesterolemia: markedly elevated low density lipoprotein cholesterol levels and severe atherosclerosis on a low-fat chow diet. Nat Med 4:934-938

30. Hammad SM, Powell-Braxton L, Otvos JD, Eldridge L, Won W, Lyons TJ (2003) Lipoprotein subclass profiles of hyperlipidemic diabetic mice measured by nuclear magnetic resonance spectroscopy. Metabolism 52:916-921

31. Klionsky DJ, Abdelmohsen K, Abe A et al (2016) Guidelines for the use and interpretation of assays for monitoring autophagy (3rd edition). Autophagy 12:1-222 
32. Iwai-Kanai E, Yuan H, Huang C et al (2008) A method to measure cardiac autophagic flux in vivo. Autophagy 4:322-329

33. Oh SH, Lim SC (2009) Endoplasmic reticulum stress-mediated autophagy/apoptosis induced by capsaicin (8-methyl-N-vanillyl-6nonenamide) and dihydrocapsaicin is regulated by the extent of cJun NH2-terminal kinase/extracellular signal-regulated kinase activation in WI38 lung epithelial fibroblast cells. J Pharmacol Exp Ther 329:112-122

34. Wu H, Wang MC, Bohmann D (2009) JNK protects Drosophila from oxidative stress by trancriptionally activating autophagy. Mech Dev 126:624-637

35. Szegezdi E, Logue SE, Gorman AM, Samali A (2006) Mediators of endoplasmic reticulum stress-induced apoptosis. EMBO Rep 7: 880-885

36. Frost LS, Mitchell CH, Boesze-Battaglia K (2014) Autophagy in the eye: implications for ocular cell health. Exp Eye Res 124:56-66

37. Remé CE, Sulser M (1977) Diurnal variation of autophagy in rod visual cells in the rat. Graefes Arch Klin Exp Ophthalmol 203:261-270

38. Remé CE, Wirz-Justice A, Rhyner A, Hofmann S (1986) Circadian rhythm in the light response of rat retinal diskshedding and autophagy. Brain Res 369:356-360

39. Piano I, Novelli E, Della Santina L, Strettoi E, Cervetto L, Gargini C (2016) Involvement of autophagic pathway in the progression of retinal degeneration in a mouse model of diabetes. Front Cell Neurosci 10:42

40. Rodriguez-Muela N, Koga H, Garcia-Ledo L et al (2013) Balance between autophagic pathways preserves retinal homeostasis. Aging Cell 12:478-488

41. Kato M, Ospelt C, Gay RE, Gay S, Klein K (2014) Dual role of autophagy in stress-induced cell death in rheumatoid arthritis synovial fibroblasts. Arthritis Rheum 66:40-48

42. Tian Y, Kuo C, Sir D et al (2015) Autophagy inhibits oxidative stress and tumor suppressors to exert its dual effect on hepatocarcinogenesis. Cell Death Differ 22:1025-1034

43. Davis RJ (2000) Signal transduction by the JNK group of MAP kinases. Cell 103:239-252

44. Verfaillie T, Salazar M, Velasco G, Agostinis P (2010) Linking ER stress to autophagy: potential implications for cancer therapy. Int J Cell Biol 2010:930509

45. Fujita E, Kouroku Y, Isoai A et al (2007) Two endoplasmic reticulum-associated degradation (ERAD) systems for the novel variant of the mutant dysferlin: ubiquitin/proteasome $\operatorname{ERAD(I)~and~}$ autophagy/lysosome ERAD(II). Human Mol Genet 16:618-629

46. Urano F, Wang X, Bertolotti A et al (2000) Coupling of stress in the ER to activation of JNK protein kinases by transmembrane protein kinase IRE1. Science 287:664-666

47. Yu L, McPhee CK, Zheng L et al (2010) Termination of autophagy and reformation of lysosomes regulated by mTOR. Nature 465 : $942-946$ 